\title{
3. ASORS-Jahreskongress
}

\section{Supportive Maßnahmen als essentielle Bausteine moderner Tumortherapien}

Bereits zum dritten Mal fand der Jahreskongress der Arbeitsgemeinschaft Supportive Maßnahmen in der Onkologie, Rehabilitation und Sozialmedizin (ASORS) der Deutschen Krebsgesellschaft statt. Die rege Beteiligung von 300 Personen aus allen Disziplinen, die mit Onkologie assoziiert sind, zeigte, dass es - wie der Vorsitzende der ASORS Professor Hans Helge Bartsch, Freiburg formulierte - «nicht nur um Strahl und Stahl geht, sondern in erster Linie um den betroffenen Patienten». In diesem Zusammenhang sind die supportiven Maßnahmen unverzichtbar, machen sie doch die onkologischen Therapien erst möglich. Dementsprechend breit war das Spektrum der Vorträge.

«Es geht zudem nicht nur um die direkten Tumortherapien, sondern die steigende Anzahl an Langzeitüberlebenden erhöht den Bedarf an onkologischen Rehabilitationsmaßnahmen», ergänzte der Rehabilitationsmediziner. Die erfreuliche Nachricht ist laut Bartsch, dass die Patienten diese Maßnahmen annehmen: Der Aufwärtstrend bei den Rehabehandlungen zulasten der gesetzlichen Rentenversicherung hat sich auch in den letzten Jahren fortgesetzt. Als Trends in der onkologischen Rehabilitationsmedizin zeichnen sich eine stärkere Ergebnisorientierung durch Qualitätssicherung und Versorgungsforschung sowie Entwicklung einer S3-Leitlinie speziell zur Rehabilitation aus. Darüber hinaus werden die Angebote immer weiter flexibilisiert, indem ambulante Angebote weiter ausgebaut werden. Zudem erfolgt eine bessere Vernetzung zwischen Akutversorgung und Rehabilitation, indem beispielsweise Modelle der Integrationsversorgung gewählt werden. Bartsch betonte: «Gerade im Umfeld der funktionierenden Vernetzung mit Betriebsärzten, Akutkrankenhäusern und Hausärzten hat sich die onkologische Rehabilitation zu einer entscheidenden Schnittstelle entwickelt.»

\section{Neutropenieprophylaxe unzureichend}

Supportive Therapien und deren Umsetzung in der alltäglichen Praxis, erfolgt noch immer nicht so, wie es im Sinn der Patienten und einer guten Versorgung wäre. Dies zeigte sich außerordentlich deutlich am Beispiel einer aktuellen Patientendokumentation, welche erfasst, wie im «therapeutischen Alltag» tatsächlich die Neutropenieprophylaxe durchgeführt wird und inwieweit die Leitlinien in der Praxis befolgt werden. In dieser retrospektiven epidemiologischen Erhebung bei den Indikationen Mammakarzinom, Bronchialkarzinom und maligne Lymphome wurden insgesamt die Daten von knapp 2000 Patienten aus Kliniken und Praxen erfasst. «Es zeigte sich, dass viele Patienten, die laut Leitlinien unbedingt eine Primärprophylaxe mit G-CSF erhalten sollten, diese nicht erhalten», kommentierte der wissenschaftliche Leiter Professor Hartmut Link, Kaiserslautern, die ersten, ausgewerteten Ergebnisse. Beispielsweise erhielten noch nicht einmal $15 \%$ aller Patienten mit einem Bronchialkarzinom, die Chemotherapieprotokolle mit einem hohen Risiko für febrile Neutropenie verabreicht bekamen, die entsprechende Neutropenieprophylaxe. Bei Patienten mit einem Mammakarzinom waren die Resultate etwas besser, aber ebenfalls noch nicht befriedigend. Die endgültigen Ergebnisse werden zum Amerikanischen Krebskongress (ASCO) vorgestellt, wobei in Zukunft noch intensiver nach den Ursachen gefragt werden muss, warum die Leitlinienempfehlungen zur Neutropenieprophylaxe nicht eingehalten werden.

\section{Umsetzungsstrategien in der Praxis}

Momentan wird die S3-Leitlinie zur Supportivmedizin erarbeitet. «Das Kick-off-Meeting ist bereits erfolgt und wir arbeiten mit allen Kräften daran, 2015 die entscheidende Konsensuskonferenz zu den Leitlinien durchzuführen», erläuterte die Federführende der ASORS-Leitlinie PD Dr. Karin Jordan, Halle. Bis dahin liegt allerdings noch ein weiter, arbeitsreicher Weg vor der Arbeitsgruppe (www.s3supportiv.de).

Wie allerdings heute schon Leitlinien als ein wichtiger Bestandteil der Qualitätssicherung in die klinische Praxis implementiert werden können, stellte Dr. Markus Thalheimer am Beispiel des Universitätskrankenhauses Heidelberg vor. Unter der Zugrundelegung der antiemetischen Leitlinien des MASCC, der NCCN sowie der ASCO wurden diese ab 2004 in eine lokale Leitlinie umgesetzt und in die laufenden Prozesse implementiert. Es erfolgte eine Anpassung der Bestellprozesse auf die Leitlinie sowie Verbrauchsmessungen und punktuelle Nachschulungen. Nachdem neue Substanzen in der Antiemese zugelassen wurden, erfolgte eine ständige Weiterentwicklung der Prozesse. Die entstandene Leitlinie wurde von AMK und KV genehmigt und direkt im Krankenhaussystem abgebildet und IT-gestützt erfasst. Die Bestell- und Applikationsdaten wurden weiterhin direkt in die Abrechnung übernommen. Thalheimer resümierte: «Wir haben nicht nur eine - hoffentlich - bessere, leitliniengerechte Versorgung unserer Patienten, sondern haben dadurch auch deutliche Einsparpotenziale identifiziert». Zukünftig sollen weitere Prozesse in der Onkologie auf diesem Weg erfasst werden.

\section{KARGER}

Fax +497614520714

Information@Karger.com

www.karger.com (c) 2013 S. Karger GmbH, Freiburg 